INTERNATIONAL JOURNAL OF SYSTEMATIC BACTERIOLOGY

Vol. 20, No. 3 July 1970

pp. $259-268$

Copyright 1970, Iowa State University Press

\title{
RANGE OF CELLULAR AND COLONLAL MORPHOLOGIES OF ACTINOMYCES ISRAELII
}

John M. Slack and Mary Ann Gerencser

Department of Microbiology, Medical Center

West Virginia University, Morgantown, West Virginia 26505

ABSTRACT. The range of cellular and colonial morphologies which occurred in a large group of A. israelii cultures, including recent isolates, from this country or abroad were studied. The strains were grown on BHI plates or in thioglycollate broth under conditions routinely used in a diagnostic laboratory. Stained smears or dark-field preparations from thioglycollate broth showed rods which varied from diphtheroid-like to long filaments. Microcolonies on BHI agar ranged from small and compact to spider-like with the latter predominating. Mature colonies on the same medium had a smooth or rough appearance. Rough colonies were found in two-thirds of the cultures. The smooth colonies were circular, convex and soft in consistency. The rough colonies were more irregularwith a granular or a highly convoluted surface, friable to hard and adherent to the medium.

\section{INTRODUCTION}

Colonial and cell morphology has been and continues to be used as the initial basis for isolation and as a major criterion for identification and differentiation of Actinomyces. An excellent early work on this subject is that of Wright (1905). Since then a number of authors (Negroni and Bonfiglioli, 1937; Rosebury et al., 1944; Howell et all., 1959; Pine et al., 1960 ; B rock and Georg, 1969) have described the distinctive morphological characteristics of the genus.

However, the range of cellular and colonial morphologies observed in a number of strains of $\mathrm{A}$. israelii, the principal human pathogen, has not been adequately described. Thus, it is the aim of this work to give in some detail these morphologies as observed on a large number of isolates from various countries and more precisely define the mor phological limits of this species. This will provide a pictorial guide to enable one to more easily identify $\underline{A}$. israelii and to emphasize that an organism having a diphtheroid morphology with little evidence of branching may well be an Actinomyces.

\section{MATERIALS AND METHODS}

Cultures. The sources' of the 64 strains used are given by Slack et al. (1969). Twenty were isolated in this country, the others in 
Australia, Argentina, France, Scotland and Germany. The biochemical and serological reactions of these organisms are also given in the same paper.

Stained and dark-field preparations. Smears were made from cultures grown in Fluid Thioglycollate B roth (Difco) for either 3 or 7 days and stained by the Gram's method (Conn, 1957). Dark-field preparations were made from the same cultures but a suspension of the organisms was washed 3 times in saline and then a drop transferred to a slide and a cover glass was forcefully pressed down onto the drop and sealed into place with nail polish. Dark-field microscopy was done using a Leitz Dl. 20 condenser and a X 95 fluorite oil objective with an iris diaphragm. Photographs were taken with the Leitz Orthomat automatic camera.

Microcolonies. Cultures were streaked onto Brain Heart Infusion (BHI) agar plates and incubated at $37^{\circ} \mathrm{C}$ in a jar containing an atmosphere of $95 \% \mathrm{~N}_{2}-5 \% \mathrm{CO}_{2}$. Microcolonies were examined at $18-24$ and $48 \mathrm{hr}$. by transferring a small section of the agar to a microscope slide and observing the colonies microscopically at X 250 and X 400 . For oil immersion examination a cover glass was dropped onto the agar surface. Photographs were taken with the Leitz Orthomat automatic camera.

Macrocolonies. BHI plates were streaked and incubated as described above. Observations were made at 7 and 14 days with a dissecting microscope at X 7 :0 X 50. Photographs were taken using an AO dissecting microscope with a photographic tube attachment. Satisfactory lighting of the colonies with reflected light was accomplished through the use either singly or in combination of two small incandescent spot lights and a fluorescent light attached to the microscope. The terminology used to describe the colonies in the main is that recommended in the Manual of Microbiological Methods (1957).

\section{RESULTS}

Gram stains. After $72 \mathrm{hr}$. growth in thioglycollate broth the predominant cellular appearance is diphtheriodal with $\mathrm{V}$ and $\mathrm{Y}$ forms (Fig. 1). The organism is gram positive and the cells may be stained intensely and uniformly (Fig. 1) but frequently the staining is irregular giving the cells a beaded appearance (Fig. 3 and 4). Branching is limited and usually occurs as Y or T forms. Cultures will, however, exhibit elongated branching filaments beginning with the initiation of growth (Fig. 2 and 3). Again, staining may be regutar and intense (Fig. 2) but it is often irregular and neither the borders nor ends of the filaments can be clearly seen (Fig. 3). Occasionally the filaments will be intertwined with terminal bulbous ends (Fig. 4). Diphtheroid cells will also have swollen or bulbous ends. The shorter, straight, stained cells (Fig. 1) ranged from $3-8$ microns in length and approximately 0.5 microns wide. The longer filaments (Fig. 2, 3 and 4) are 10-40 microns or more in length.

Dark-field preparations. Dark-field microscopy is an excellent method for determining cell morphology and was used for the examina tion of growth in broth or on solid media. Again the predominant cell morphology was that of the irregularly shaped rod exhibiting short $V$ or $Y$ branching (Fig. 5 and 6). The degree of branching varied considerably 


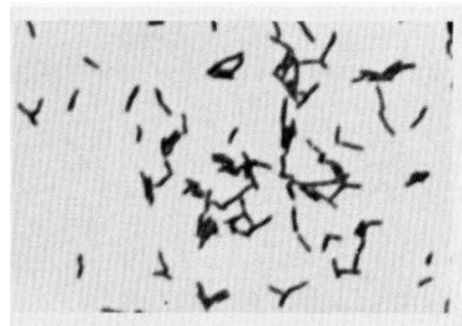

FIG. 1. Short rods, fragmention, $\mathrm{Y}$ forms. X 1580

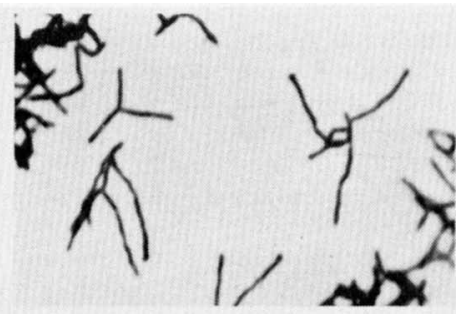

FIG. 2. Longer filaments with branching, more solidly stained. $\times 1580$

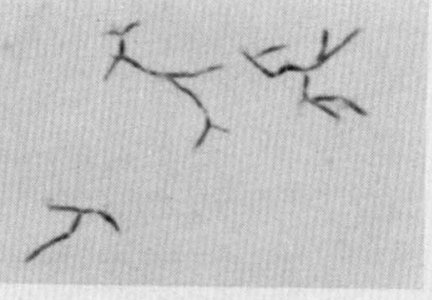

FIG. 3. Branching filaments staining lightly and irregularly, very common appearance. X 1260

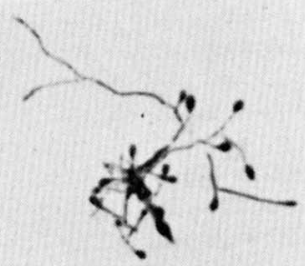

FIG. 4. Branching, irregularly staining filaments, some of which have a bulbous end. Not frequent ly found. X 1580

Figures 1-4. Gram stains of A. israelii after 72 hours of growth in thioglycollate broth. 
with shorter branching rods (Fig. 6) being observed more frequently than long filaments. These could be seen in all cultures although at times they were found only after many fields had been examined. The elongated multiply-branching filaments (Fig. 6) were more frequently observed if the growth in broth was quite granular. Figure 8 clearly shows the formation of a very small colony with branching filaments. The wavy appearance of the one extending filament is often seen.

As expected, the individual cells and filaments appeared to be larger under dark-field than in the stained smears. The cell width varied from 0.8-1.2 microns and the small non-branching cells (Fig. 5) were from 3 microns to 12 microns in length. The branching filaments varied considerably in length with the one in Fig. 6 being 30 microns long.

Microcolonies. The culture streaked on BHI plates were first examined microscopically after $18-24 \mathrm{hr}$. of incubation. In most instances, the pattern of microcolony formation could be established at this time with one-third of the cultures forming small compact colonies (Fig. 9). The se colonies may increase in size and exhibit little evidence of branching filaments or the cells will elongate with more branching to produce a larger but still somewhat compact colony (Fig. 10). The remaining two-thirds of the cultures produced microcolonies made up of filaments showing elongated multiply-branching filaments (Fig. 11) although sometimes the filaments would be shorter and have a more angular type of branching, The "spider" colony (Fig. 12) which often appears to arise from a central point is commonly described as the characteristic colony of this species. Observations were repeated at $48 \mathrm{hr}$. but the basic colony shape seen at $24 \mathrm{hr}$. did not change except that the colonies were larger, more compact and less useful for species differentiation. The individual cells (Fig. 9) varied from 3-10 microns in length and those as in Fig. 11 were 30-40 microns in length. The large colony in Fig. 10 was approximately 30 microns in diameter.

Mature colonies. Mature colonies of A. israelii are illustrated in figs. 13-18. These colonies varied from smooth to rough and after 14 days ranged in size from $0.5 \mathrm{~mm}$ to $2.0 \mathrm{~mm}$ with an occasional larger colony. After 7 days of incubation the colony type was established and usually the only change with further incubation was in size; however, surface convolutions (Fig. 18) may become more pronounced in older colonies.

Approximately 22 cultures produced predominately smooth colonies (Fig. 13). Forty-two strains produced predominately rough colonies of the types shown in Figs. 16-18. A given culture may produce primarily smooth or rough colonies although sametimes both could be seen on a single plate. For example, a single culture might form smooth (Fig. 13), rough (Fig. 17) and bread-crumb colonies (Fig. 18) on the same plate and subcultures from any one of these colonies would again produce a similar variety of colonies.

Of the smooth colony types, the one shown in Fig. 17 is most frequently observed and may be described as: circitar to slightly irregular, convex to pulvinate, smooth, entire, opaque, white to graywhite and soft. Variations include (a) differences in opacity which coupled with slight, irregular indentations give the smooth surface a textured look (Fig. 14); (b) differences in elevations producing colonies 


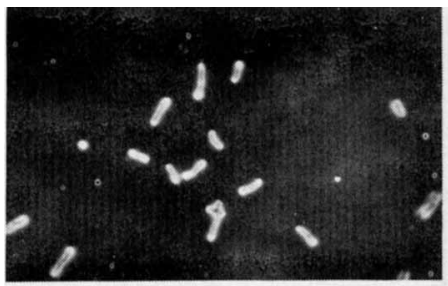

FIG. 5. Short rods, irregular shapes, not suggestive of an actinomycete. X 950 .

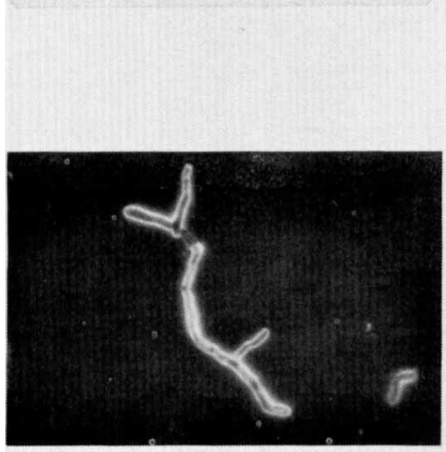

FIG. 7. An elongated branching filament which is $30 \mu$ long and varies from $0.8-1 \mu$ in width. X 1580 .

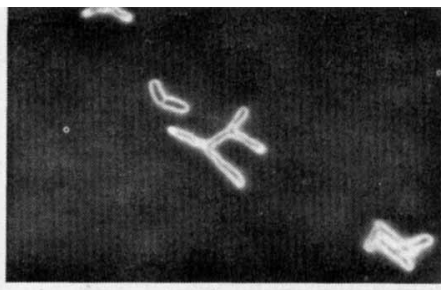

FIG. 6. One filament with distinct branching and a shorter element that may be dividing. X. 1580

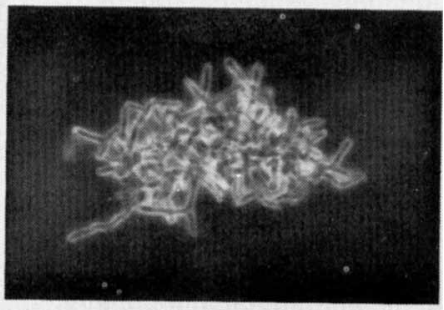

FIG. 8. A microcolony with branching filaments, onei of which shows the rather common wayey appearance. X 1580.

Figures 5-8. Dark-field preparations from $17 \mathrm{hr}$ cultures $A$. israelii in thioglycollate broth. 


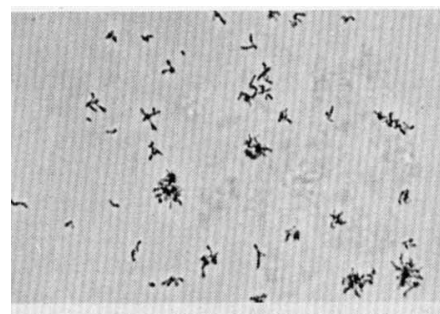

FIG. 9. Sma11, compact colonies beginning to form. $\times 410$

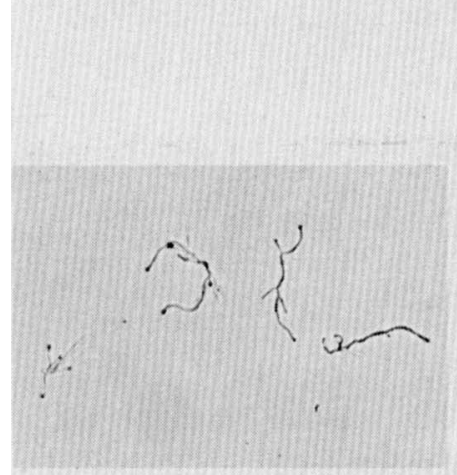

FIG. 11. Elongated, multiple branching filaments. $\times 410$

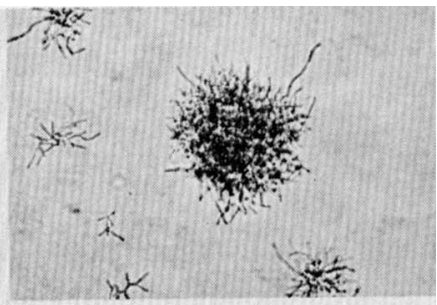

FTG, 10. Larger, more compact colony of brauching filaments with one much longer filament extending from the colony. $\times 410$ branching filaments seemingly growing out from the center. X 410

Figures 9-12. Microscopic examination of unstained $24 \mathrm{hr}$ microcolonies of $\mathrm{A}$. israelii growing on $\mathrm{BHI}$. 


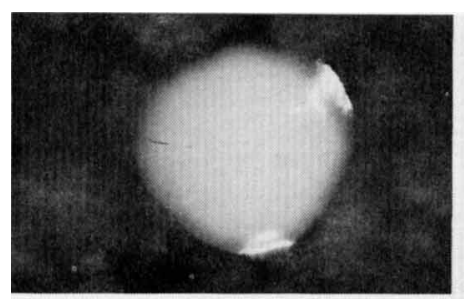

FIG. 13. Smooth, convex entire edge. X 20

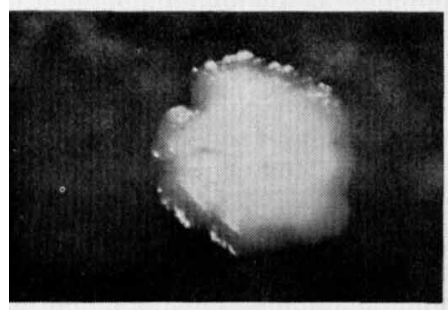

FIG. 15. Rough, convex, finely granular surface; opaque center with translucent edge. X 40

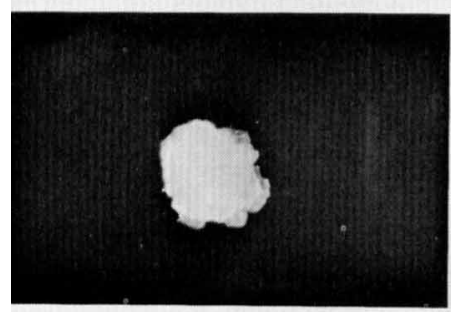

FIG. 17. Rough, heaped, smooth surface with convolutions. X 20

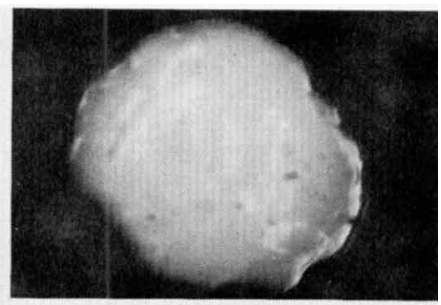

FIG. 14. Smooth, textured surface, convex; irregular colony with entire edge. $\times 40$

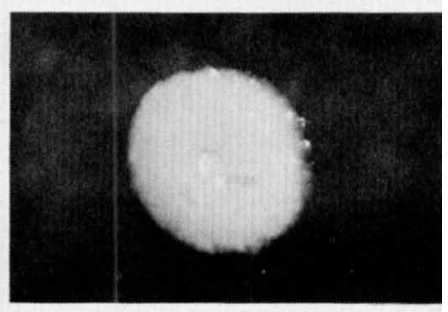

FIG. 16. Rough, convex with depressed center; finely granular surface. X 40

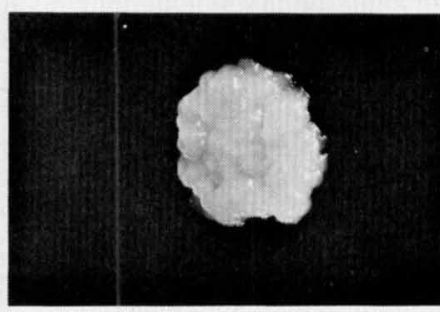

FIG. 18. Rough, heaped with highly convoluted surface (bread-crumb colony). X 20

Figures 13-18. Microscopic examination of 14-day macrocolonies of A. israelii growing on BHI. 
that are umbonate, convex with shallow rather broad depressions or convex with one or more central grooves.

There were two main types of rough colonies. The first of these with its different appearances (Fig. 15 and 16) may be described as: circular to irregular, convex to pulvinate, finely granular to granular (ground-glass type of surface), erose to lobate, grayish-white, opaque and friable. These colonies could have depressed centers (Fig. 16). While not actually dull, they were less shiny than the smooth colonies and the other type of rough colonies. The second type of rough colony varies in appearance (Fig. 17 and 18 ) and is described as: circular to irregular, pulvinate to heaped, undulate to lobate, white, opaque, shiny, hard and adherent to the medium. The surface convolutions vary in depth causing the colonies to vary in appearance from almost smooth (Fig. 17) to bread-crumb like (Fig. 18). Such colonies may have central depressions varying from slight to those deep enough to appear as holes (Fig. 18). Some rough colonies have a narrow, translucent edge (Fig. 17) around the opaque, convex to pulvinate center. Less frequently observed are colonies with a wide opaque, slightly convoluted edge with an umbonate central area which is flat on top.

\section{DISCUSSION}

In cellular and colonial morphological descriptions of the Actinomyces, the terms filamentous, branching, molar-toothed are encountered as depicting characteristics associated with the species of this genus. Except for the work of Howell et al., (1959) there is little information on the range of cellular or colonial morphologies occurring in a given species grown under the conditions most frequently used for isolation and identification.

This paper describes the various morphologies observed in the study of sixty-four isolates of A. israelii. The cultures represented fresh isolates, those maintained in laboratories twenty or more years, and isolates from a number of countries.

A. israelii produced filaments of varying lengths exhibiting primary and secondary branching. Filaments could be demonstrated in stained smears or in wet preparations of any given culture, however, the predominant cell morphology was often that of short rods which were irregularly shaped and showed $\mathrm{V}, \mathrm{Y}$, or $\mathrm{T}$ branching. These rods were usually 3-10 microns in length and 0.8-1.2 microns in width in dark field preparations. Stained smears of such cultures often showed cells with irregular staining and an occasional palisade arrangement. These were often indistinguishable from Corynebacterium. The growth of these short rods into filaments and the subsequent fragmentation of filaments into such rods was shown by Erikson (1940). When cultures are isolated showing this type of morphology it is important to consider that the organism may be an Actinomyces.

Dark-field microscopy, apparently little used at the present time, is a rapid and useful technique for studying cell morphology. We use such preparations directly from broth or solid media cultures in pre ference to stained smears. With high dry magnification the entire area of the preparation can be rapidly scanned and the presence of relatively short branching filaments determined. 
Ordinarily, the most helpful morphological criterion in identifying an Actinomyces is the type of microcolony produced after $18-24 \mathrm{hr}$. of incubation on BHI. Such an early observation is essential because even within $48 \mathrm{hr}$. the colonies become more entire and hemispherical and lose some of their differential characteristics. In the majority of instances the early microcolony of $\mathrm{A}$. israelii is the easily recognized, striking "spider" type (Fig. 12). Otherwise the colony may be made up of filaments showing angular branching or less frequently of short rods with a minimum of branching. With the se colonies a single filament extending from the growth is often seen (Fig. 10).

As the colonies mature, evidence of branching filaments is lost. They become more rounded and show varying degrees of surface irregularity from umbonate to convolutions with deep crevices and depressions. A crater-like cavity frequently develops in the center of the colony (Fig. 18). However, some cultures form smooth hemispherical colonies providing no indication that the organism is an Actinomyces.

In $\underline{A}$. israelii, both micro and macrocolonies may appear rough or smooth and any given culture may produce a variety of morphological types sometimes on the same plate. Subcultures of any of the se colony types may again give rise to different shapes and sizes. It has not been possible to show serological or physiological differences between subcultures from a rough or a smooth colony thus, this does not appear to be an $S$ - $R$ variation as exhibited in the Enterobacteriaceae.

The range in cellular and colonial morphologies shown for $\underline{A}$. israelii is characteristic of the Actinomyces but the factors that promote such changes have not been determined. Jordan and Howell (1965) studied some nutritional factors which affected morphology and Pine and Boone (1967) compared cell wall analyses with morphological forms. The range of morphological types seen in our work was obtained from cultures growing on the same medium and under the same conditions of incubation so presumably nutritional factors were not a major factor in the observed variation.

In identifying unknown cultures, the genus Actinomyces is considered for a gram positive, anaerobic to facultatively anaerobic organism with branching filaments and a rough colony. But this genus should also be considered for gram positive, facultative anaerobes which produce only diphtheroidal cells and smooth colonies until such identification is specifically excluded.

\section{ACKNOW LEDGEMENTS}

This investigation was supported in part by Public Health Services grant AI-01801 from the National Institutes of Allergy and Infectious Diseases.

The assistance of Sandra Landfried is gratefully acknowledged. 


\section{LITERATURE CITED}

Brock, D. W. and L. K. Georg. 1969. Determination and analysis of Actinomyces israelii serotypes by fluorescent antibody procedures. J. Bacteriol., 97:581 -588 .

Conn, H. J. 1957. Manual of Microbiological Methods. p. $16,149$. McGraw-Hill Book Company, New York.

Erikson, D. 1940. Pathogenic anaerobic organisms of the Actinomyces group. Brit. Med. Res. Council. Spec. Rep. Ser. No. 203.1-63.

Howell, A., W. C. Murphy, F. Paul, and R. M. Stephan. 1959. Oral strains of Actinomyces. J, Bacteriol, , 78:82 -95.

Jordan, H. V. and A. Howell. 1965. Nutritional control of cellular morphology in an aerobic actinomycete from the hamster. J. Gen. Microbiol. 38:125-130.

Negroni, P., and H. Bonfiglioli. 1937. Morphology and biology of Actinomyces israelii. J. Trop. Med. Hyg. 40:226-232. 240-245

Pine, L. and C. J. Boone, 1967. Comparative cell wall analyses of morphological forms within the genus Actinomyces. J. Bacteriol. 94:875-883.

A. Howell, and S. J. Watson. 1960. Studies of the morphological physiological and biochemical characters of Actinomyces bovis. J. Gen. Microbiol., 23:403-424.

Rosebury, T., C. J. Epps, and A. R. Clark. 1944. A study of the isolation, cultivation and pathogenicity of Actinomyces israelii recovered from the human mouth and from actinomycosis in man. J. Infect. Diseases. 74:131-149.

Slack, J. M., S. Landfried, and M. A. Gerencser. 1969. Morphological, biochemical and serological studies on 64 strains of Actinomyces israelii. J. Bacteriol., 97:873-884.

Wright, J. H. 1905. The biology of the microorganism of actinomycosis. J. Med. Res. 13:349-404. 